\title{
La politique de l'Eau, organisation et gestion : rapport général
}

\author{
Water policy, organization and management : general report
}

par J.P. Poly

Agence de l'Eau Adour-Garonne

Water policy, organization and management is a wide and complex area which is rapidly changing. The papers presented at the 25 th Congress en Hydraulics deal with different aspects of policy and managnement.

\section{I 口 INTRODUCTION}

Il s'agit là d'un domaine extrêmement vaste et complexe et qui fait l'objet aujourd'hui d'évolutions rapides et importantes (PJ. : communication du ministre de l'Aménagement du Territoire et de l'Environnement au Gouvernement le 20 mai 1998 et communiqué de presse du 22 juillet 1998). Ses frontières vont des grands principes qui, depuis 30 ans, ont largement façonné la politique de l'eau dans notre pays, aux conditions de la gestion locale auxquelles sont confrontés divers opérateurs, comme les communes, en passant par l'action des services de l'Etat. Au cœur de ce domaine, l'articulation des dispositions réglementaires et opérationnelles et la coordination des acteurs qui les ont en charge constituent le nœud de la gestion intégrée d'une ressource rare et vulnérable.

Les rapports particuliers suscités en ce domaine par les 25 èmes journées de l'hydraulique, organisées par la Société Hydrotechnique de France, abordent, chacun, certains aspects de l'organisation et de la gestion de l'eau et leur analyse nous conduit à une présentation du sujet rappelant d'abord les grands principes qui ont guidé l'action publique en France depuis 30 ans, montrant ensuite comment des efforts partagés de planification et de programmation viennent donner corps à ces principes, comment une gestion équilibrée prolonge cette planification grâce à des opérateurs comptables de la gestion locale de la ressource en eau, pour situer enfin le système français dans un contexte communautaire et mondial.

\section{II $\square$ LES GRANDS PRINCIPES DE LA POLI- TIQUE DE L'EAU EN FRANCE ET LEUR MISE EN CEUVRE DE 1964 À NOS JOURS}

Ils sont en général bien connus et ont été à maintes occasions présentés. Faisant aujourd'hui l'objet d'un examen cri- tique, nous nous attacherons à considérer leurs transformations passées et leurs perspectives d'évolution.

Deux dates, 1964 et 1992, ont profondément marqué le cadre de la politique de l'eau dans notre pays.

En 1964, le législateur a donné un tour radicalement nouveau à la politique de l'eau en France en mettant en avant les trois principes novateurs :

- reconnaissance d'une nouvelle circonscription de gestion, le bassin hydrographique, se substituant, en la matière, aux découpages administratifs traditionnels ;

- à l'intérieur de cette entité naturelle, planification et gestion concertée et intégrée pour dépasser le cadre traditionnel de la gestion sectorielle ;

- taxation des pollutions déversées, des prélèvements et consommations d'eau.

La loi de 1964 et ses décrets d'application ne se sont pas contentés d'afficher ces principes tout à fait novateurs pour l'époque, ouvrant ainsi la voie aux futurs grands chantiers de la décentralisation. Pour leur donner corps, deux institutions ont été mises en place : le Comité de Bassin, assemblée délibérante, véritable Parlement de l'eau dans le bassin, et l'Agence Financière de Bassin (aujourd'hui Agence de I'Eau), établissement Public de l'Etat, instrument d'intervention technique et surtout financier.

Ces trois grands principes ont été mis en application grâce :

- aux compétences dévolues aux Agences sur la circonscription hydrographique de leur bassin ;

- à la représentation des différentes catégories d'utilisateurs de la ressources en eau dans les instances de bassin, Comité de Bassin et Conseil d'Administration de l'Agence, clef de la concertation ;

- à la gestion financière autonome de l'Agence dans le cadre de ses programmes d'intervention quinquennaux.

C'est selon ces dispositions que les Agences et les Comités ont pu élaborer et exécuter six programmes d'intervention se 
traduisant par des investissements importants et reposant sur une concertation approfondie au sein de la communauté des usagers du bassin.

Malgré une stagnation de leurs moyens financiers pendant une douzaine d'années due à la lutte contre l'inflation et à la maîtrise par le Gouvernement des prélèvements obligatoires, alors même que la population française augmentait et que la valeur ajoutée industrielle croissait, les Agences ont contribué à une amélioration de la qualité des eaux en faisant reculer les pollutions organiques et toxiques.

En 1996, les objectifs de qualité, fixés au début de la décennie 80 , étaient atteints sur $45 \%$ des points de mesures, $90 \%$ de la population française permanente ou saisonnière était desservie par un réseau de collecte public et $85 \%$ raccordée à une station d'épuration. Au cours des dix dernières années, le rendement des ouvrages s'est accru et de nouvelles formes de pollution ont été prises en compte.

Il est trop tôt pour mesurer l'impact de l'effort encore accru accompli entre 1992 et 1996 (Vlème Programmes des Agences de l'Eau se traduisant en l'espace de 5 ans par un doublement du taux des redevances). Dès 1994, la France avait achevé la transcription de la Directive dite " Eaux usées " et. durant cette période, les Agences ont contribué au financement de plus de 51 milliards de Francs de travaux d'assainissement pour les communes.

Mais les problèmes évoluent et de nouvelles formes de pollution apparaissent. La qualité des grands cours d'eau s'est améliorée mais celle des petites rivières s'est encore dégradée. La pollution d'origine agricole impose de nouvelles approches. Nos concitoyens s'étonnent et quelquefois ne comprennent pas l'augmentation du prix de l'eau mais. dans le même temps, demandent une protection plus forte encore de leur environnement. La réglementation européenne devient toujours plus contraignante.

\section{- 2.1 Le bassin versant hydrographique}

On conçoit que, dans ces conditions, le bassin versant hydrographique soit bien devenu le cadre d'une gestion technique des interdépendances hydrauliques, mais c'est aussi le lieu d'expression des solidarités financières qu'il convient de développer pour atteindre les objectifs de ressource et de qualité fixés d'un commun accord entre les usagers.

M. Christian BERNAD a choisi de porter témoignage de la démarche entreprise par les usagers de la vallée du Lot pour développer la gestion de cette ressource dans une perspective d'aménagement du territoire.

Cet exemple doit sans doute conduire nos organismes de bassin à s'appuyer d'avantage sur les institutions interdépartementales en charge de l'aménagement de nos grands fleuves et à développer dans leurs prochains programmes une approche territoriale bien à l'écoute des initiatives locales et soucieuse d'un aménagement équilibré et harmonieux du territoire du bassin.

Partant du Livre Blanc de 1970, débouchant sur les Assises de l'Eau de 1990, en passant par les Schémas d'Aménagement de Bassins prescrits par la circulaire de 1978, la planification par bassins hydrographiques n'a jamais cessé de se développer durant les 30 dernières années.

En Adour-Garonne, elle a abouti bien souvent à la création d'institutions par grands sous-bassins : Institutions Interdépartementales pour l'Adour, le Lot, la Charente, Syndicat Mixte d'Etude et d'Aménagement de la Garonne, Etablissement Public pour la Dordogne.
Les diagnostics et les propositions techniques que cette planification a suscités et la concertation qui l'a accompagnée ont constitué une expérience irremplaçable pour les communautés d'usagers. Mais la faiblesse de cette planification tenait à son caractère peu contraignant.

Un grand tournant devrait être pris avec la loi de 1992 rendant obligatoire une planification dans chaque bassin avec les Schémas Directeurs d'Aménagement et de Gestion des Eaux (SDAGE) et déterminant les conditions de son éventuel prolongement par des Schémas d'Aménagement et de Gestion des Eaux (SAGE), même si la mise en œuvre de ces derniers reste subordonnée à la volonté des acteurs locaux.

Dans leur rapport " La gestion concertée et décentralisée de l'eau en France ". MM. JOUSSEAUME et RETKOWSKY, après avoir rappelé certaines définitions, mettent l'accent sur certains points essentiels du dispositif.

Ils insistent à juste titre sur l'un des aspects trop souvent négligés et encore peu connus de ceux qui ne sont pas au cœur du dispositif : l'évaluation de la politique de l'eau.

Il convenait de mettre en avant ce souci d'évaluation, pour deux raisons. D'abord il n'est pas encore si fréquent que les conditions d'évaluation de l'action publique soient dès le départ prévues. C'est d'ailleurs dans cette faille que se sont engouffrés, l'an passé, la Cour des Comptes et le Commissariat au Plan pour remettre en cause certaines pratiques ou dénoncer certaines dérives de la politique de l'eau mises en œuvre par nos organismes de bassin.

MM. JOUSSEAUME et RETKOWSKY nous montrent en fait l'importance des efforts récemment accomplis par le ministère de l'Environnement et les Agences de l'Eau en ce domaine. Un tableau de bord central permettra ainsi de suivre la mise en œuvre des principales mesures préconisées par les SDAGE. De même, les Agences de l'Eau disposent désormais de tableaux de bord mesurant le niveau d'exécution de leurs programmes d'intervention.

Au-delà du progrès que représente la mise à disposition de ces outils, il convient d'insister sur l'importance de l'appareil statistique progressivement mis en place avec :

- les différents réseaux de mesures regroupés au sein du Réseau National des Données sur l'Eau (RNDE) et l'observatoire des zones humides :

- les données sur les redevances et les primes dont disposent les Agences, instruments de mesure des pollutions et des efforts d'épuration :

- les caractéristiques des ouvrages aidés par les Agences de l'Eau.

Les auteurs rappellent que le SDAGE constitue désormais le cadre de l'action publique et fonde sur le principe de compatibilité les interventions de chacun dans le domaine de la gestion de la ressource. Dans ces conditions, les programmes d'intervention des Agences, notamment, se placent au service des objectifs fixés par ces Schémas.

Enfin, nos rapporteurs abordent les conditions d'une gestion concertée locale avec le SAGE et le contrat de rivière ou de baie.

La loi sur l'eau de 1992 propose, en effet, une nouvelle démarche de planification locale (SAGE), là où les efforts consentis pour préserver une ressource très menacée n'ont pas été suffisants. Quant au contrat de rivière, il constitue une procédure bien éprouvée, appelée à prolonger au plan opérationnel la mise en ceuvre des SAGE. Ces deux outils complémentaires se relayent donc sur le terrain. 
Le rapport a le mérite de mettre en application la méthode d'évaluation en présentant un bilan des procédures SAGE et contrat de rivière.

Elle montre à l'évidence que le train est en marche et que, sur bien des points, il conviendra encore de coordonner et d'ajuster ces procédures.

Ne doit-on pas ainsi considérer que le SDAGE est directeur, comme l'indique son nom ? Il encadre les décisions publiques et les programmes de l'Etat et des collectivités qui, dans le domaine de l'eau, devront être compatibles. Le SDAGE remplit-il pour autant les conditions de cette convergence des actions réglementaires de l'Etat, financières des Agences, politiques et financières des collectivités locales? On peut se poser la question si l'on estime que seule la planification concertée débouche sur ces convergences.

Il appartient aux SAGE de réunir ces conditions. Or, la loi ne fait que proposer cette procédure. L'initiative locale reste déterminante. Mais en fait, bien des mesures du SDAGE peuvent être mises en œuvre sans recourir aux SAGE...

Il est donc vraisemblable que les objectifs du SDAGE ne seront pas atteints par la seule procédure des SAGE mais par une série d'outils et d'initiatives variés dont la coordination sera sans doute difficile et laborieuse.

\subsection{La concertation}

Encore plus certainement qu'il y a 30 ans, la concertation est la clef du succès d'une politique publique de l'eau dans une société sans cesse plus exigeante où le débat démocratique est de règle pour la gestion d'une ressource dont on mesure mieux aujourd'hui la rareté et la vulnérabilité et qui fait l'objet de sollicitations de plus en plus diversifiées et importantes.

Par le passé, nos organismes de bassin ont déjà su répondre aux évolutions de la société et leur composition a été récemment encore adaptée (représentation des grandes villes, représentation des institutions interdépartementales et des irrigants en Adour-Garonne).

Les rapports de MM. SICARD et CAMEO-PONZ témoignent de la participation de deux catégories de partenaires exigeants ou critiques. Le Directeur de l'Agence de l'Eau Adour-Garonne ne peut que partager les déclarations de M. SICARD dont le rapport souligne les conditions et perspectives d'une meilleure intégration du monde agricole dans les programmes des organismes de bassin que souhaite développer nos autorités de tutelle.

Il décrit le long chemin semé d'embûches parcouru ensemble. M. SICARD met l'accent sur le contrat de gestion d'un bassin qu'il connaît bien, celui de la Charente, avec l'Institution Interdépartementale pour l'Aménagement du fleuve Charente et de ses affluents.

Il rappelle aussi les récentes évolutions d'une politique de gestion équilibrée de la ressource plus contractuelle entre l'Agence et le monde agricole qui repose sur un accord cadre portant majoration des redevances et sur des accords particuliers avec chaque Chambre d'Agriculture favorisant la mise en œuvre d'interventions circonstanciées faisant évoluer les pratiques culturales.

Je connais les critiques adressées à cette démarche : trop timorée, trop accommodante, pas assez entreprenante... Peutêtre faut-il faire observer à nos détracteurs que, si les agriculteurs sont aujourd'hui pleinement impliqués dans la politique de l'eau, c'est bien aux instances de bassin qu'on le doit, Conseils d'Administration et Comités de Bassin, qui ont été parfois bien seuls face à ces partenaires difficiles. M. SICARD donne la condition essentielle de cette prise de responsabilisation : la transparence.

Le monde associatif a toujours été représenté dans les Comités de Bassin et il a pris au fil du temps une place grandissante dans le débat. L'importance de cette contribution a été reconnue par le législateur avec la création de la Commission du Milieu Naturel Aquatique, indépendante des instances de bassin et jouissant d'une certaine autonomie par rapport à elles.

Sans doute faut-il s'attendre demain à une participation encore renforcée du monde associatif à la définition des programme d'aménagement et de gestion des eaux avec sa représentation au sein des Conseils d'Administration des Agences.

\section{- 2.3 La taxation des usagers}

Révolution engagée par la loi de 1964, la taxation des usagers n'est pas rentrée dans les faits en un tour de main et sans que quelques arrangements avec le principe pollueur/payeur soient recherchés. Certains trouvent là aujourd'hui matière à tergiverser.

L'important n'est-il pas qu'au bout de 30 ans le principe pollueur/payeur ne soit pas resté seulement un slogan, que les usagers aient contribué effectivement au financement des programmes des Agences de l'Eau en fonction des perturbations occasionnées sur la ressource et que, par leurs représentants, ils aient décidé eux-mêmes des conditions d'allocation des sommes recueillies ? L'essentiel, aussi, n'estil pas qu'un système de perception de ces redevances, avec ses règles, ses outils statistiques, sa jurisprudence, ait été mis en place?

Néanmoins, à force d'adaptation pour prendre en compte de nouvelles atteintes à l'environnement, le système est devenu compliqué et difficile à appréhender. Il doit donc évoluer afin d'être plus équitable, plus simple, plus transparent (cf. communication du ministre).

On sait depuis longtemps que certaines catégories d'usagers dont les activités perturbent gravement les écosystèmes, sont restées en marge du système. La redevance Modification du Régime des Eaux, dont la mise en place est aujourd'hui envisagée et qui doit interpeller notamment les promoteurs de grands aménagements et les extracteurs de granulats, devrait conférer aux Agences la légitimité d'intervenir plus résolument afin de prévenir les inondations et certains désordres hydrologiques et apporter à ces établissements les moyens financiers correspondants.

De même, ainsi que je l'ai déjà indiqué, le traitement de la pollution d'origine agricole, en France comme dans les autres pays, reste un objectif primordial et difficile. Il exige de nouvelles approches.

Mettre en avant les principes novateurs de la loi de 1964 et les organismes spécifiques chargés de les mettre en œuvre ne doit pas conduire à passer sous silence l'organisation administrative traditionnelle dont le rôle reste important et déterminant pour le développement d'une politique de l'eau aux plans national et régional.

Il est peu de pays où cette organisation soit simple : quand on cherche à présenter l'organisation française, on suscite la perplexité. Je n'essaierai donc pas de le faire. Mais je voudrais souligner quelques points essentiels :

- La complexité administrative française découle en 
grande partie d'un encombrement institutionnel évident : commune, département, région. A chaque niveau, l'Administration doit être organisée.

- Elle découle aussi de la nature même de l'élément en jeu, l'eau. Tous les ministères sont peu ou prou concernés. D'où les structures de coordination : Mission Interministérielle de l'eau au plan national, Comité technique de l'eau au niveau régional. Coordination également entre usagers au plan national comme dans les Comités de Bassin avec le Comité National de l'Eau.

- La circonscription de bassin est venue s'ajouter à ce découpage. D'où les formes de liaisons nécessaires :

- tutelle du ministère de l'Environnement sur les Agences de l'Eau :

- Préfet coordonnateur de bassin :

- DIREN de bassin.

\section{III — LA GESTION OPÉRATIONNELLE}

On donnerait une vision très incomplète de l'organisation de la gestion de l'eau et des acteurs qui s'y emploient si l'on se contentait de présenter ces grands principes de la gestion de l'eau, la composition et le fonctionnement des instances de bassin ou les procédures et objectifs des Schémas d'Aménagement et de Gestion des Eaux.

La gestion de l'eau s'analyse selon diverses échelles de temps et d'espace. Elle épouse le territoire et son histoire. Elle se construit en permanence grâce à l'intervention de nombreux acteurs comme les Conseils Généraux, les communes et leurs groupements, les acteurs économiques et techniques comme les SAR et EDF. Différents rapports particuliers témoignent de cette grande diversité de la gestion opérationnelle.

Au premier rang des opérateurs, il faudrait citer les communes, responsables de ce qui reste essentiel pour nos concitoyens : l'alimentation en eau potable et l'assainissement.

Aucun rapport ne traite de ce rôle pourtant fondamental. Mais le Directeur d'Agence peut témoigner combien les communes sont les partenaires incontournables d'une politique de reconquête de la qualité des ressources en eau. Une politique contractuelle (contrat d'agglomération) a par exemple été mise en place entre les communes urbaines et les Agences.

Les responsabilités des communes sont aujourd'hui très grandes, confrontées qu'elles sont à l'application des normes européennes, devant négocier avec les grands groupes, l'affermage ou les concessions, et tenues d'être attentives aux réactions de l'opinion devant l'augmentation du prix de l'eau.

Cette responsabilité est abordée sous un autre angle par deux rapports, celui de M. BOURRELIER, " Les parties prenantes de la gestion des crues " et celui de MM. TALIERCIO, LE TRIONNAIRE, BOUYSSES, ODIER, ROY et de Mme GODON qui traite de l'amélioration de la prévision locale des inondations et du rôle des communes.

M. BOURRELIER aborde largement la question de la participation intégrée des différents acteurs pour la gestion des risques de crues. Il indique que l'évaluation de la politique publique met en évidence la nécessité d'une clarification entre les fonctions de l'Etat et celles des collectivités territoriales à différents niveaux, et aussi l'importance de l'implication de ces collectivités. C'est précisément une telle implication que relate le deuxième rapport sur la prévision locale des crues et le rôle des communes.

Il s'agit d'adapter l'information et les prévisions émanant des services de l'Etat aux caractéristiques locales. Cette démarche aboutit à l'élaboration d'outils permettant aux communes de prévoir les crues et inondations à partir des informations transmises. En retour, la connaissance, sur le terrain, des épisodes passés et les observations de la population locale peuvent enrichir les modèles généraux de prévision des services de l'Etat.

Cette expérience menée en lle-de-France ouvre, me semble-t-il, des perspectives qui devraient être discutées et approfondies. En effet, dans le cas des inondations, elle cherche à faire de la commune un acteur autonome et responsable participant à sa propre protection, collaborant à l'élaboration des méthodes et des outils de prévention. Imaginons le capital de bonnes volontés, de bon sens, de recommandations avisées que l'on pourrait mobiliser auprès de la population si on prenait le temps de rechercher la collaboration de cette dernière dans d'autres domaines de la gestion des crues. Ainsi sont illustrées de nouveau les vertus de la concertation/participation.

Les Conseils Généraux aussi sont des acteurs importants. Leur rôle vis-à-vis des petites communes rurales est décisif et ils sont devenus, surtout après les lois de décentralisation, des partenaires essentiels des Agences de l'Eau, par le biais de conventions et contrats départementaux pour la bonne gestion des ressources en eau de nos zones rurales.

Un certain nombre de rapports émanent d'opérateurs économiques et techniques importants. II s'agit d'Electricité de France et des Sociétés d'Aménagement Régional. CACG, $\mathrm{SCP}$ et BRL. Leur histoire les conduit à être aujourd'hui des acteurs confrontés aux impératifs d'une gestion équilibrée de la ressource en eau alors que leur mission principale originelle est d'ordre économique. Pour cette raison, leur expérience et leurs réflexions sont fort instructives.

Le rapport de M. CASANOVA " La politique de gestion de l'eau à Electricité de France " met d'emblée l'accent sur cette mission économique et sur les obligations de Service Public de cette entreprise. II montre le rôle de I'hydroélectricité dans ce service.

Après ce rappel tout à fait capital, M. CASANOVA explique comment cette contrainte de gestion hydroélectrique doit aller de pair avec la prise en compte aujourd'hui des autres usages de l'eau, sous l'effet de l'évolution du contexte externe (externe à EDF! le langage trahit).

Il me semble, en fin de compte, qu'EDF est sans doute aujourd'hui l'organisme le plus nettement engagé, de façon très opérationnelle, dans cette gestion équilibrée dont on se contente en fait, la plupart du temps et pour le moment, de parler. Les exemples cités par M. CASANOVA le prouvent.

Mais ce qui est aussi intéressant, c'est qu'EDF tente de se forger une doctrine, dont M. CASANOVA présente les grands axes, sur lesquels je voudrais revenir :

- concertation avec tous les acteurs concernés :

- contractualisation :

- indemnisation :

- maîtrise d'ouvrage réalisant les arbitrages ;

- études scientifiques notamment économiques sur la valeur des différents usages de l'eau.

Ces deux derniers points sont particulièrement importants. La concertation, la contractualisation, les arbitrages inter- 
viendront si un maître d'ouvrage se déclare. M. CASANOVA compte sur les collectivités et leurs Ententes (Institutions Interdépartementales ou Syndicats). II s'appuie ce faisant sur son expérience dans le bassin Adour-Garonne où existent, le long de presque tous les grands fleuves, des Ententes de ce type (Adour, Charente, Lot, Dordogne, Garonne). Mais encore faudrait-il sans doute que ces collectivités renforcent leurs moyens techniques pour devenir de véritables partenaires, autonomes et fiables à la fois, comme le souhaite M. CASANOVA.

L'appréciation de la valeur des différents usages peut sembler théorique. Elle est pourtant au cœur des arbitrages. EDF sait affecter un prix à un $\mathrm{m}^{3}$ d'eau valorisé au plan énergétique et on ne peut donc lui reprocher de s'interroger sur ce que devient cette valeur quand le $\mathrm{m}^{3}$ est lâché pour une compétition de Canoë-Kayak. Comment valorisons-nous au mieux notre ressource hydraulique dans la gestion combinée de ses différents usages ? Je pense que nous devrions nous mobiliser pour définir sinon un modèle comparable à celui d'EDF du moins une démarche conjuguant économie et approches socio-politiques, nous permettant, sur un territoire donné, d'optimiser la gestion de l'eau.

A partir d'une expérience différente et plus locale, M. VILLOCEL montre comment une société d'aménagement régional, la Compagnie d'Aménagement des Coteaux de Gascogne (CACG) a été conduite, elle aussi, dans le même contexte économique et social et à l'occasion d'épisodes de sécheresse sévères, à faire évoluer ses méthodes et à reconsidérer son rôle, pour devenir ce que M. VILLOCEL nomme un opérateur de la gestion de l'eau.

Comme celui de M. CASANOVA, le rapport de M. VILLOCEL est passionnant parce qu'il témoigne de l'interrogation d'une institution sur son rôle et du regard qu'elle porte sur l'évolution permanente de son environnement. J'ai choisi d'en souligner deux aspects susceptibles d'être repris dans les discussions.

La gestion de l'eau par quota volumétrique pour l'irrigation constitue certainement une contribution décisive à la gestion intégrée mise en œuvre par la compagnie après les sécheresses de 1989 et 1990 . On retrouve là les ingrédients déjà mentionnés dans d'autres rapports mais qui prennent ici un caractère opérationnel évident :

- la transparence, évoquée par M. SICARD dans son rapport, trouve ici son expression avec la mise en place d'instruments de mesure et la constitution de fichiers des prélèvements, le suivi des consommations des quotas et le relevé des infractions aux contrats :

- la concertation, au sein d'une commission où sont analysées les contraintes de gestion : cohérence globale, file d'attente, acceptation des restrictions :

- la réglementation et sa compatibilité avec les interventions du gestionnaire :

- enfin l'existence d'un opérateur qui a développé ses outils techniques et sans lequel on peut se demander si une telle gestion peut devenir aussi effective et aussi rigoureuse.

Je livre ici cette interrogation pour que nos rapporteurs s'en saisissent.

La réflexion sur le rôle d'opérateur de la gestion de l'eau joué par une SAR rejoint celle de M. CASANOVA sur la place d'EDF dans la gestion combinée des usages.

M. VILLOCEL note tous les atouts des SAR : la complémentarité de leurs deux objectifs principaux, l'hydraulique et le développement rural, qui leur donne une vision territoriale, l'association de l'efficacité de la gestion privée et de la durabilité du service public, la technicité donnant confiance aux élus locaux et régionaux.

On peut se demander, comme le suggère M. VILLOCEL, si une partie de la réponse à la recherche par M. CASANOVA d'un maître d'ouvrage, maître de l'arbitrage politique exercé sur des bases techniques et scientifiques, ne réside pas dans le développement d'une collaboration entre des collectivités et des SAR.

La gestion de la ressource ne se résume pas toutefois à l'expérience relatée par M. VILLOCEL qui note lui-même que cette gestion reste difficile à engager sur les bassins dépourvus de ressources artificielles. La gestion de l'eau ne se confond pas en effet avec la gestion d'ouvrage et il faudrait sans doute que les SAR accroissent leurs capacités techniques dans le domaine de la maîtrise de la qualité des eaux, de la protection et de la sauvegarde des écosystèmes pour pouvoir jouer un rôle opérationnel plus large dans la gestion intégrée des eaux.

Les réflexions de M. VILLOCEL sont judicieusement complétées par la présentation du projet franco-espagnol d'aqueduc Languedoc-Roussillon/Catalogne par MM. BLANC et IMBERT de BRL. Cet ambitieux projet qui pourrait modifier la répartition des eaux entre deux grandes régions méditerranéennes, témoigne, de façon probante, du rôle important que peut jouer une Société d'Aménagement Régional aux plans des études techniques préalables, des montages juridiques et financiers puis de l'exploitation d'un grand ouvrage.

MM. PLANTEY et REIG relatent une expérience identique. Les ouvrages que la société du Canal de Provence a en charge sont encore plus importants qu'en Gascogne.

\section{IV — LE SYSTÈME FRANÇAIS DANS L'EXPÉ- RIENCE INTERNATIONALE : PLACE ET PERSPECTIVES}

La Société Hydrotechnique de France a intitulé ses 25èmes journées " L'école française de l'eau au service du développement mondial ".

Je me trouve donc logiquement conduit à conclure mon intervention, en essayant de situer l'organisation que je viens de caractériser de façon bien schématique, par rapport à certaines expériences étrangères. Je le ferai sur la base des rapports produits par quelques collègues que je tiens à remercier d'avoir accepté de rechercher aujourd'hui avec nous les principes d'organisation les plus aptes à répondre aux défis que doit relever la gestion de l'eau. On verra combien la convergence est importante dans des situations pourtant très différentes, tributaires de la géographie, façonnées par l'histoire, modelées par la culture.

Elle est si importante que, sous l'impulsion française et après la Conférence de Rio en 1992, s'est constitué le Réseau International des Organismes de Bassin (RIOB) qui fait l'objet d'un rapport de mon collègue CHIROUZE, Directeur de l'Agence de l'Eau Rhône-Méditerrannée-Corse.

Que 68 organismes de 32 pays sur 4 continents s'entendent sur une déclaration mettant en exergue quelques-uns des principes les plus importants ayant fondé la politique de l'eau en France depuis 30 ans montre au moins que notre pays a su adopter assez tôt des principes dont la pertinence est aujourd'hui reconnue par une large communauté. 
Le gouvernement du Québec a signé, le 21 mars 1996 à Mexico, son adhésion à la charte du RIOB. M. LATULIPPE nous montre dans son rapport quel mouvement est en cours au Québec pour fonder une nouvelle politique de l'eau. La gestion par bassin versant en sera sans doute un axe essentiel. Une opération pilote est en cours sur la rivière Chaudière, avec la création d'un Comité de Bassin, l'établissement d'un Schéma Directeur et la proposition d'un modèle de financement fondé sur la redevance.

En opérant le rapprochement avec le système de bassin qui prévaut en France, plusieurs questions se posent :

- une Agence financière dotée d'une autonomie de gestion sera-t-elle constituée pour la rivière Chaudière ou les redevances perçues seront-elles versées au budget gouvernemental pour être ensuite redistribuées ?

- le Conseil d'Administration de CORBARIC II réunira-til les représentants de toutes les catégories d'usagers (élus, acteurs socio-économiques, associations, fonctionnaires) ?

- enfin, COBARIC II doit proposer les mécanismes de conciliation et d'harmonisation du Schéma Directeur de l'eau avec les pouvoirs municipaux et les schémas d'aménagement. J'ai déjà indiqué que dans notre pays la question ne me semblait pas complètement résolue et nous serons donc intéressés de savoir dans quelles voies nos cousins québécois comptent s'engager pour parvenir à cette conciliation.

Placé dans des conditions géographiques, économiques et culturelles sensiblement différentes, M. JELLALI, de la Direction Générale de l'Hydraulique du Maroc, relate une expérience qui doit être rapprochée de celle de M. LATULIPPE. On retrouve à l'origine une loi récente visant à réformer la politique de l'eau en mettant notamment en avant l'organisation d'une gestion par bassin hydrographique et le principe pollueur/payeur.

L'approche est progressive et déjà se profile la mise en place d'une Agence sur le bassin de l'Oum Er Rabii, dans lequel l'activité économique, surtout agricole, est importante. Tous les aspects d'une telle opération ont été traités : programmation financière, réglementation, politique d'information, formation de l'encadrement.

Les questions posées à M. LATULIPPE peuvent être adressées à M. JELLALI et des réponses croisées seraient intéressantes dans la discussion. M. JELLALI laisse notamment entrevoir certaines difficultés pour la perception des redevances, difficultés que nous connaissons bien en France.

Les Agences de l'Eau de la Pologne qui, pour la plupart, ont passé des accords de coopération technique avec les Agences de l'Eau françaises, participent activement aux instances du RIOB (Réseau International des Organismes de Bassin).

Comme nous l'explique M. BADOWSKI, Directeur Général de l'Agence de l'Eau de la Moyenne Vistule, les organismes de bassin de la Pologne se sont d'ores et déjà dotés de Comités de Bassin pour mieux organiser la concertation entre les usagers et se sont attelés à la préparation de Schémas Directeurs d'Aménagement et de Gestion des Eaux afin d'orienter l'action publique à moyen terme.

Il n'y a pas de doute sur le fait que le rapport de M. SEAGER sur les " Perspectives actuelles et futures de l'organisation et de la gestion de l'eau au Royaume-Uni " est celui qui nous conduit à nous interroger le plus fortement sur les vertus et les faiblesses de notre système.

La gestion par bassin versant, en tant que principe fondamental de la gestion de l'eau, bénéficie d'une longue expérience au Royaume-Uni. Mais le système paraît plus centralisé que le nôtre. Une Agence de I'Environnement met en œuvre actuellement un programme de 133 plans des Agences locales de l'Environnement, qui couvre l'ensemble des bassins versants d'Angleterre et du Pays de Galle. Contrairement à ce qui se passe ou se prépare en France avec la mise en œuvre des SAGE, la démarche est systématique, ce qui ne semble pas nuire à la prise en compte des réalités de terrains et à l'implication des partenaires locaux et du public en général.

L'un des aspects intéressants du système anglais réside dans le contrôle financier des sociétés privées d'eau à la suite de la privatisation des services de l'industrie de l'eau, opérée par le Bureau des Services de l'Eau. Il serait intéressant que M. SEAGER développe ce point.

Enfin, le système en vigueur au Royaume-Uni peut paraître mieux équilibré, plus réaliste que chez nous, en reposant sur une planification négociée entre le Bureau des Services de l'Eau (pour les consommateurs), les sociétés privées (pour les actionnaires) et l'Agence pour l'environnement (pour l'environnement).

On comprend comment ce système permet l'augmentation des investissements destinés au traitement des eaux usées domestiques. Mais une série d'éclaircissements mériteraient d'être apportés : qu'en est-il de la lutte contre la pollution industrielle ? de la maîtrise de la pollution agricole ? du financement des autres actions permettant de maîtriser la ressource, de la restaurer et de la protéger ?

Comme on le constate, la politique de l'eau en France, comme dans bien des régimes du monde, doit se fonder sur quelques principes intangibles :

- Elle doit être concertée et relativement autonome afin de permettre aux usagers de l'eau de définir les objectifs de qualité et de ressource qu'ils se fixent pour leur bassin et déterminer les moyens qu'ils se donnent pour les atteindre.

- Elle doit être équilibrée entre les mesures opérationnelles et les mesures réglementaires, entre le respect des milieux et le développement des activités économiques, entre les différents usages de l'eau.

- Elle doit être intégrée afin de conjuguer gestion de la ressource et aménagement du territoire, afin de combiner les approches sectorielles et afin de respecter les continuités amont/aval et transversales de nos cours d'eau. 